\title{
Influence of Contamination on Diffusion of Point Defects in Rutile Reduced in Vacuum*
}

\author{
By Eisuke Iguchi** and Kazuo Yajima***
}

\begin{abstract}
Single crystals of rutile in the form of slices were reduced in vacuum to investigate the diffusion of point defects. The reduction was performed in three kinds of vacuum atmospheres by using (A) a diffusion pump without traps, (B) a diffusion pump with liquid nitrogen traps and (C) an ion pump. The process of reduction is considered to represent the process of diffusion of point defects induced by reduction. The diffusion coefficients of point defects in the reduced rutile were determined by a theory of diffusion in a plane sheet with an equal surface concentration and a uniform initial distribution of solute in a sheet. These coefficients obtained in vacuum by a diffusion pump varied with the reduction time and this tendency was more remarkable in the case of (A). But the diffusion coefficients obtained in the case of $(\mathrm{C})$ were nearly constant at any reduction temperature independent of the reduction time except for the beginning stage. It appears that the differences are due to the contamination introduced by the diffusion pump. By using the ion pump, the diffusion coefficient of point defects in the reduced rutile can be expressed as
\end{abstract}

$$
D=91 \exp [-(2.42 \pm 0.33)(\mathrm{eV}) / k T] \mathrm{cm}^{2} / \mathrm{sec} .
$$

This activation energy is very close in value to that of oxygen ions which diffuse in rutile by the vacancy mechanism. (Received July 17, 1971)

\section{Introduction}

The crystal structure of rutile $\left(\mathrm{TiO}_{2}\right)$ is tetragonal. In the stoichiometric state, it is an insulator but changes into n-type semiconductor when reduced slightly ${ }^{(1)(2)}$. This means that point defects introduced by reduction act as donor centers. The nature of the point defects have so far been studied in many ways, but much remains unclear.

It is said that there exist two kinds of point defects in the reduced rutile, because two types of reducing reactions are thought to be possible. These are

$$
\mathrm{TiO}_{2} \rightarrow \mathrm{TiO}_{2(1-x)}+x \mathrm{O}_{2}^{\uparrow}+2 x \mathrm{O}_{\mathrm{v}}^{2+}+4 x e^{-}
$$

and

$$
\mathrm{TiO}_{2} \rightarrow(1-x) \mathrm{TiO}_{2}+x \mathrm{O}_{2}^{\uparrow}+x \mathrm{Ti}_{1}^{4+}+4 x e^{-},
$$

where $\mathrm{O}_{\mathrm{V}}, \mathrm{Ti}_{\mathrm{I}}$ and $e^{-}$are an oxygen vacancy, an interstitial titanium ion and an electron, respectively.

According to eq. (1), the point defects are oxygen vacancies and their valences change from $+2,\left(\mathrm{O}_{\mathrm{v}}^{2+}\right)$, to $0,\left(O_{v}^{0}\right)$, by trapping two electrons. Therefore $0_{v}^{0}$ can become donor centers by releasing two electrons per vacancy.

According to eq. (2), the point defects are interstitial titanium ions and their valences change from $\mathrm{Ti}_{I}^{4+}$ to $\mathrm{Ti}_{\mathrm{I}}^{m+}(0 \leqq m<4)$ by trapping electrons. So $\mathrm{Ti}_{I}^{m+}$ can

* This paper was published in Japanese in J. Japan. Inst. Metals, 35 (1971), 395.

** Department of Metallurgical Engineering, Faculty of Engineering, Yokohama National University, Yokohama, Japan.

*** Graduate School, Yokohama National University, Yokohama, Japan.

(1) R. R. Hasiguti : Metal Phys., 9 (1963), 95.

(2) F. A. Grant : Rev. Mod. Phys., 31 (1959), 646.

(3) P. F. Chester : J. Appl. Phys., 32 (1961), 2233.

(4) J. H. Becker and W. R. Hosler : Proc. Int. Conf. on Crystal Lattice Defect, Tokyo and Kyoto, J. Phys. Soc. Japan, Suppl. II, 1963, p. 152.

(5) R. R. Hasiguti, N. Kawamiya and E. Yagi : Proc. 7 th Int. Conf. Phys. Semiconductors, Paris, 1964. Dunod, Paris, 1965, A 1255 . become donor centers by releasing $(4-m)$ electrons per interstitial titanium ion. Though the exact value of $m$ is unknown, many papers interpret their experimental results taking $m$ as $3^{(3) \sim(7)}$. In this case, however, $3 m x$ electrons should remain as excess electrons, which can hardly be explained.

There are a number of papers which support the existence of $\mathrm{O}_{\mathrm{V}}{ }^{\left({ }^{(8)} \sim(10)\right.}$, and of $\mathrm{Ti}_{\mathrm{I}}{ }^{(8) \sim(7)}$, but only a few papers ${ }^{(11)(12)}$ show the coexistence of $\mathrm{O}_{\mathrm{V}}$ and $\mathrm{Ti}_{\mathrm{I}}$.

In these studies of the reduced rutile, the atmosphere in which $\mathrm{TiO}_{2}$ is reduced becomes a problem of great importance. For instance, when reduced in $\mathrm{H}_{2}$ gas, the hydrogen ion is observed to diffuse into the specimen ${ }^{(13)}$. And it is also known that the signals of E.P.R. change with atmospheres used ${ }^{(2)(3)(11)(12)}$.

We have investigated the diffusion of point defects in rutile during the process of reduction, and found that reduction atmospheres used affect sensitively the processes of diffusion. To investigate the influences of surface contamination on the reduction of specimens, vacuum atmospheres are prepared in three different ways.

(A) Using a diffusion pump without cold traps.

(B) Using a diffusion pump with two sets of liquid nitrogen traps.

(C) Using an ion pump to keep perfectly away from contamination due to the diffusion pump oil.

In this paper, we attempted to find the best method

(6) J. H. Becker and W. R. Hosler : Phys. Rev., 137 (1965), A1872.

(7) R. N. Blumenthal, J. Coburn, J. Baukus and W.M. Hirthe : J. Phys. Chem. Solids, 27 (1965), 643.

(8) T. Hurlen : Acta Chem. Scand., 13 (1959), 365.

(9) K.S. Förland : Tionde Nordiske Kemistmotet, Stockholm, (1959).

(10) P. Kofstad : J. Phys. Chem. Solids, 23 (1962), 1579.

(11) R.R. Hasiguti, E. Iguchi and S. Takahashi : Proc. 9 th Int. Conf. Phys. Semiconductors, Moscow, 1968, Nauka, Leningrad II (1968), 1142.

(12) E. Yamaka and R. G. Barnes : Phys. Rev., 135 (1964), A 144.

(13) A von Hippel, J. Kalnajs and W. B. Westphal: J. Phys. Chem. Solids, 23 (1962), 779. 
for investigation of the diffusion kinetics of point defects in rutile without influences of surface contamination under high vacuum.

\section{Basis for Analyzing Experimental Results}

The reduction in $\mathrm{TiO}_{2}$ is considered to proceed with the diffusion of point defects introduced in the crystal. When $\mathrm{TiO}_{2}$ is reduced at a temperature $T$ and under an oxygen partial pressure $P_{\mathrm{O}_{2}}$, at first oxygen molecules are dissociated from the specimen and then point defects are introduced at the surface. These point defects may migrate into the specimen due to the larger concentration at the surface than in the inner part of the specimen. Finally the distribution of point defects becomes homogeneous in the specimen and the equilibrium concentration depends upon $P_{\mathrm{O}_{2}}$ in the reduction atmosphere.

Then, we make the following three assumptions to make this problem clear.

Assumption (1): Since the single crystals used are in the form of very thin slices, we consider only the diffusion along the direction normal to the sliced surface, ignoring that from other directions.

Assumption (2): The concentration of point defects on the surface is considered to be always constant because it is equilibrated with a constant value of $P_{\mathrm{O}_{2}}$ above the surface of the specimen.

Assumption (3) : The concentration of point defects in the specimen is initially zero before reduction.

Crank has solved the problem of the diffusion under these conditions. According to his explanation ${ }^{(14)}$, the relation between $M_{t}$ and $M_{\infty}$ is given as

$$
\begin{aligned}
M_{t} / M_{\infty}= & 1-\left(8 / \pi^{2}\right) \sum_{n=0}^{\infty} \exp \left[-D(2 n+1)^{2} \pi^{2} t / 4 h^{2}\right] / \\
& (2 n+1)^{2},
\end{aligned}
$$

where $M_{t}$ denotes the total amount of point defects which has entered into the specimen at a time $t$ and $M_{\infty}$ denotes that at $t=\infty . \quad D$ is the diffusion coefficient and $n$ is an integer.

On the other hand, the average concentration $n_{t}$, of point defects in the specimen at a time $t$ is related with the specific resistivity $\rho_{t}$, at a time $t$ by the equation ${ }^{(15)}$,

$$
1 / \rho_{t}=\alpha n_{t} e \mu,
$$

where $e, \mu$ and $\alpha$ indicate the electron charge, the electron mobility and a constant depending on the valence of defects, respectively. And $n_{t}$ is given as follows :

$$
n_{t}=M_{t} / 2 l d h
$$

where $l, d$ and $h$ are the length, the width and half a thickness of the specimen, respectively. Then we can obtain the following relation, setting the specific resistivity at $t=\infty$ as $\rho_{\infty}$ :

(14) J. Crank: The Mathematics of Diffusion, Oxford, (1956) p. 45.

(15) V.C. Nelsond and R.J. Friauf : J. Phys. Chem. Solids, 31 (1970), 825.

$$
\begin{aligned}
& \left(\rho_{\infty} / \rho_{t}\right)=\left(M_{t} / M_{\infty}\right) \\
& =1-\left(8 / \pi^{2}\right) \sum_{n=0}^{\infty} \exp \left[-D(2 n+1)^{2} \pi^{2} t / 4 h^{2}\right] /(2 n+1)^{2} .
\end{aligned}
$$

$M_{t}$ increases to $M_{\infty}$ with advance in reduction. This corresponds to the decrease of $\rho_{t}$ toward the saturated value $\rho_{\infty}$ with the increase of $t$.

In this experiment $\rho_{t}-t$ curves were plotted under a constant vacuum and at a fixed temperature. The saturated value $\rho_{\infty}$ in each of these curves was then determined so as to obtain the relation between $\left(\rho_{\infty} / \rho_{t}\right)$. and $t$. Using eq. (6), we calculated the diffusion coefficient $D$ at each time $t$. The vacuum was considered to be proportional to the oxygen partial pressure.

\section{Experimental Procedure}

A single crystal of the stoichiometric rutile grown by the Verneuil method was supplied from Nakazumi Crystal Co., Ltd. The nominal purity of the crystal was $99.99 \%$. The specimens were cut in the form of thin slice from the boule by a diamond cutter. The orientation of the slice was as follows: the plane is perpendicular to the a-axis and the longitudinal axis is parallel to the c-axis. The thickness of specimens was changed in the range from 0.4 to $1.7 \mathrm{~mm}$ depending on the conditions of reduction.

Two sets of vacuum systems were used. One is equipped with a diffusion pump and the other is an ion pump with a sorption pump and a titanium getter ion pump. Reductions of specimens were performed in the three kinds of atmospheres by the use of

(A) a diffusion pump without traps (about $1 \times 10^{-5}$ $\mathrm{mmHg}$, (B) a diffusion pump with two sets of liquid nitrogen traps (about $1 \times 10^{-5} \mathrm{mmHg}$ ) and $(\mathrm{C})$ an ion pump $\left(4 \times 10^{-9} \mathrm{mmHg}\right)$.

Hereafter we call the experiments done in $A, B$ and $C$ atmospheres as $A, B$ and $C$ series respectively.

The procedure of reduction is as follows. Specimens are set in a quartz tube connected to the vacuum system and the vacuum is kept at a specified value. Then the tube is inserted into an electrical furnace being heated to a reduction temperature $T$. After a time $t$ of the reduction, the furnace is moved away from the quartz tube as fast as possible and the specimens are cooled rapidly to room temperature. After the specimens are taken out from the vacuum system, gold wires are soldered with indium on the surface and the resistivity is measured by the four-point method using a potentiometer at room temperature. After measurements of the resistivity, the lead wires are cut off from the specimens and indium on the surface is dissolved in fusing nitric acid, then the specimens are cleaned in a solution of distilled water, acetone and ethyl alcohol. After washing, the specimens are reset in the vacuum system and the reduction is further carried on. In this way, we can obtain the changes in specific resistivity $\rho_{t}$ versus the reduction time $t$. 


\section{Experimental Results}

The specific resistvity of specimens reduced at $800^{\circ} \mathrm{C}$ in $\mathrm{A}$ series, and at $900^{\circ} \mathrm{C}$ and $1100^{\circ} \mathrm{C}$ in $\mathrm{B}$ series is shown in Fig. 1 as a function of reduction time. In Fig. 2, some curves in $\mathrm{C}$ series are indicated. Other curves in each series show similar behaviours to those in Figs. 1 and 2. In each of these curves, the specific

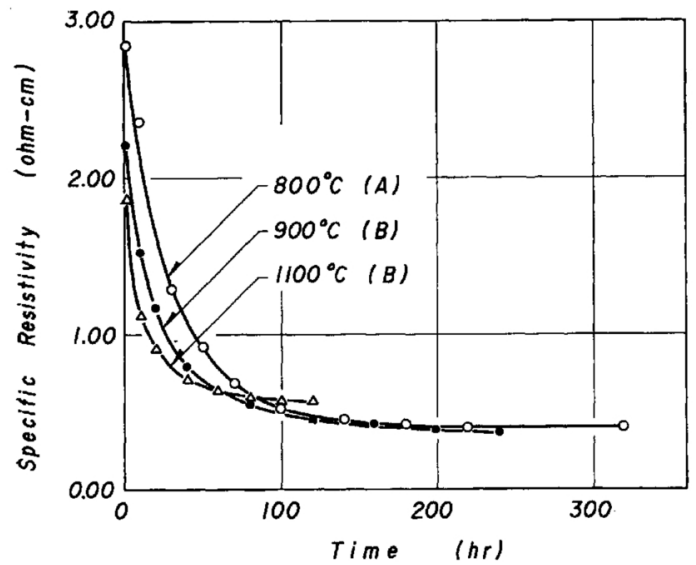

Fig. 1 Specific resistivity of specimens reduced at $800^{\circ} \mathrm{C}$ in $\mathrm{A}$ series, at $900^{\circ} \mathrm{C}$ and $1100^{\circ} \mathrm{C}$ in $\mathrm{B}$ series as a function of reduction time.

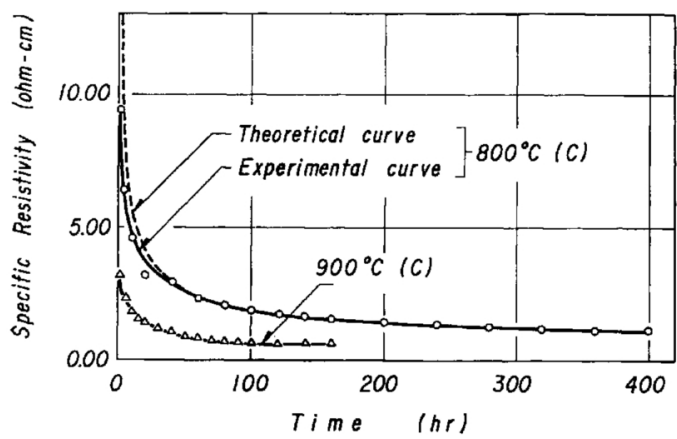

Fig. 2 Experimental and theoretical value of resistivity of a specimen reduced at $800^{\circ} \mathrm{C}$ and experimental values of a specimen reduced at $900^{\circ} \mathrm{C}$ in $\mathrm{C}$ series as functions of reduction time. The solid lines are experimental curves and the broken line is the theoretical one which is derived from eq. (6) with $D=4.64 \times$ $10^{-10} \mathrm{~cm}^{2} / \mathrm{sec}$ and $\rho_{\infty}=1.1207 \mathrm{ohm}-\mathrm{cm}$.

resistivity $\rho_{t}$ is decreasing toward a saturated value with the increase of the reduction time $t$. Under the saturated states, the concentrations of point defects are homogeneous in specimens, so that the saturated resistivities correspond to $\rho_{\infty}$ mentioned in section II.

Diffusion coefficient $D$ at a time $t$ is obtained as follows. At first $\left(\rho_{\infty} / \rho_{t}\right)$ is computed for each value of $\sqrt{D t / h^{2}}$ using eq. (6). On the other hand, $\left(\rho_{\infty} / \rho_{t}\right)$ can be obtained from the experiments with an accuracy of $1 \times 10^{-3}$. So, the summation on the right hand side of eq. (6) must be considered from $n=0$ to $n$ which gives a larger value than $10^{-3}$ for $\left(8 / \pi^{2}\right) \exp \left[-D(2 n+1)^{2}\right.$ $\left.\pi^{2} t / 4 h^{2}\right] /(2 n+1)^{2}$. For example, the calculated values of $\left(\rho_{\infty} / \rho_{t}\right)$ for $\sqrt{D t / h^{2}}=0.250$ are listed in Table 1 . In this way, a theoretical curve of $\left(\rho_{\infty} / \rho_{t}\right)$ versus $\sqrt{D t / h^{2}}$ can be obtained in the range $\sqrt{D t / h^{2}}=0.010 \sim 1.100$. In Fig. 3 a part of the computed curve is plotted. Then,
Table $1\left(\rho_{\infty} / \rho_{t}\right)$ for $\sqrt{D t / h^{2}}=0.250$.

\begin{tabular}{c|c}
\hline \hline$n$ & $\left(8 / \pi^{2}\right) \exp \left[-D(2 n+1)^{2} \pi^{2} t / 4 h^{2}\right] /(2 n+1)^{2}$ \\
\hline 0 & 0.6956 \\
1 & 0.0227 \\
2 & 0.0007 \\
\hline
\end{tabular}

$$
\begin{aligned}
& 1-\sum_{n=0}^{\infty}\left(8 / \pi^{2}\right) \exp \left[-D(2 n+1)^{2} \pi^{2} t / 4 h^{2}\right] /(2 n+1)^{2} \\
& =1-(0.6956+0.0227+0.0007+\cdots \cdots \cdots \cdot) \\
& =0.2810 \cdots \cdots \cdots \\
& \left(\rho_{\infty} / \rho_{t}\right)=\left(M_{t} / M_{\infty}\right)=0.2810
\end{aligned}
$$

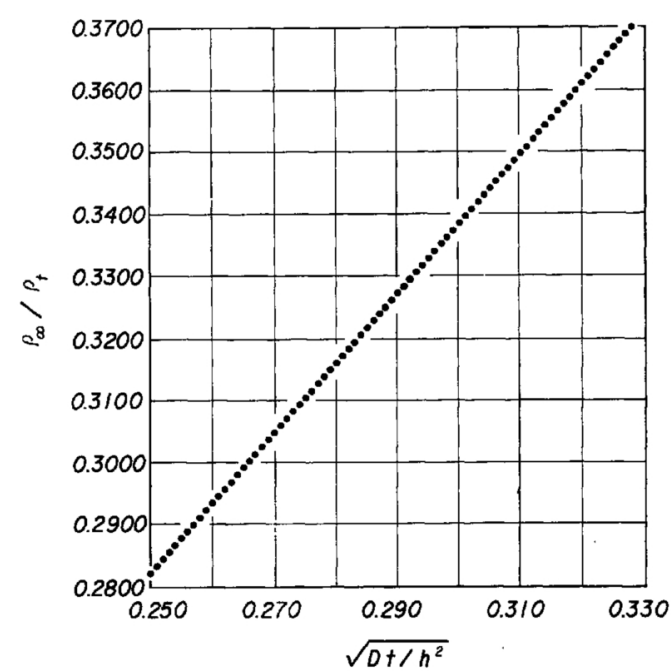

Fig. 3 Computed $\left(\rho_{\infty} / \rho_{t}\right)$ values versus $\sqrt{D t / h^{2}}$.

Table 2 Diffusion coefficient of a specimen reduced at $1000^{\circ} \mathrm{C}$ in $\mathrm{C}$ series, computed from eq. (6) with measured values $\left(\rho_{\infty} / \rho_{t}\right)$.

\begin{tabular}{r|c|c|c|c}
\hline $\begin{array}{c}t \\
(\mathrm{hr})\end{array}$ & $\begin{array}{c}\rho_{t} \\
(\mathrm{ohm}-\mathrm{cm})\end{array}$ & $\left(\rho_{\infty} / \rho_{t}\right)$ & $\sqrt{D t / h^{2}}$ & $\begin{array}{c}D \times 10^{8} \\
\left(\mathrm{~cm}^{2} / \mathrm{sec}\right)\end{array}$ \\
\hline 1 & 45.613 & 0.30526 & 0.271 & 4.46 \\
3 & 33.550 & 0.41502 & 0.368 & 2.75 \\
5 & 27.518 & 0.50600 & 0.449 & 2.45 \\
7 & 23.521 & 0.59198 & 0.528 & 2.52 \\
9 & 21.181 & 0.65738 & 0.591 & 2.36 \\
11 & 19.275 & 0.72228 & 0.659 & 2.40 \\
14 & 17.817 & 0.78150 & 0.744 & 2.41 \\
17 & 16.635 & 0.83703 & 0.806 & 2.33 \\
20 & 15.735 & 0.88496 & 0.890 & 2.41 \\
24 & 15.141 & 0.91962 & 0.968 & 2.39 \\
28 & 14.132 & 0.98528 & & \\
32 & 13.981 & 0.99592 & & \\
36 & 13.927 & & & \\
40 & 13.921 & & \\
\hline
\end{tabular}

where $2 h=9.36 \times 10^{-2} \mathrm{~cm}$ and $\rho_{\infty}=(1.3927+1.3921) / 2$ $=1.3924 \mathrm{ohm}-\mathrm{cm}$

by using an experimental value of $\left(\rho_{\infty} / \rho_{t}\right)$ at a given time $t, \sqrt{D t / h^{2}}$ is obtained. Thus the $D$ value can be determined because $t$ and $h$ are already known. In Table 2 are shown the calculated $D$ values of the specimen reduced at $1000^{\circ} \mathrm{C}$ in $\mathrm{C}$ series.

The diffusion coefficients $D$ calculated are shown in Fig. 4 for A and B series and in Fig. 5 for C series. As shown in Fig. 4, the $D$ values change remarkably as a function of reduction time at each temperature in $A$ and $\mathrm{B}$ series, but the $D$ values in $\mathrm{C}$ series are almost constant except for an early stage of the reduction as shown in Fig. 5. The constant $D$ values in the latter are considered to represent the real diffusion coefficient 


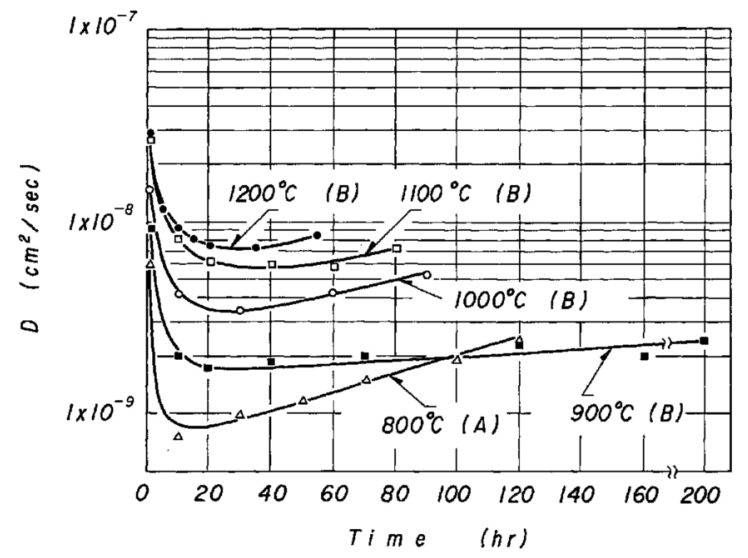

Fig. 4 Diffusion coefficient of $\mathbf{A}$ and $\mathbf{B}$ series versus reduction time, calculated from eq. (6) with the $\rho_{t}-t$ curves.

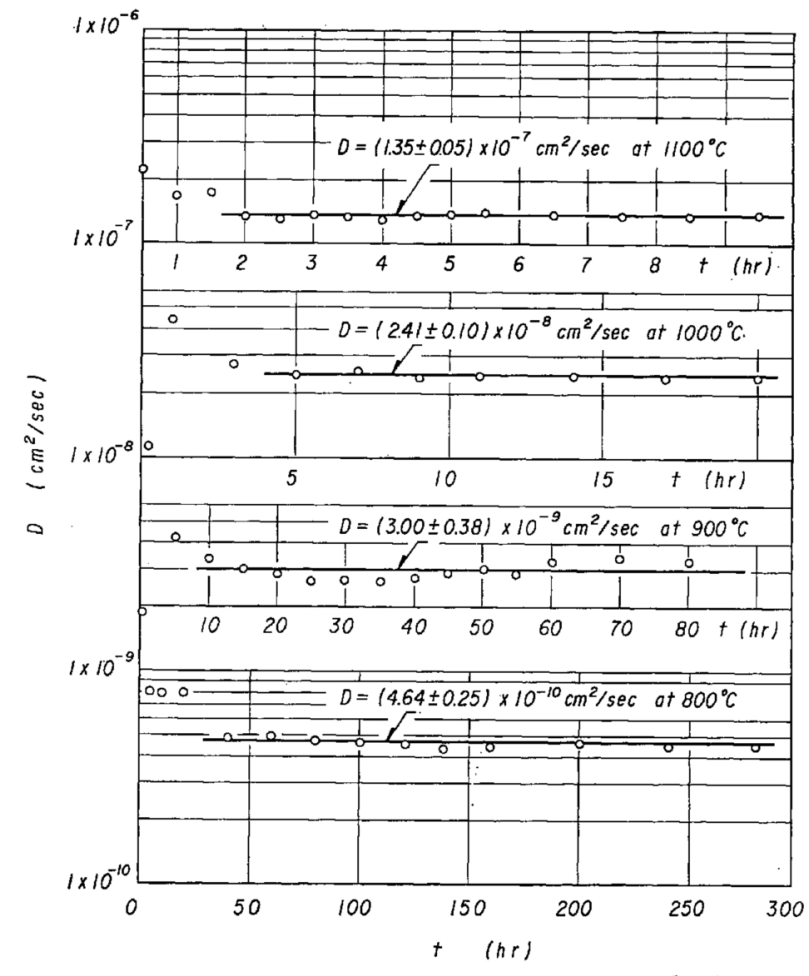

Fig. 5 Diffusion coefficient of $\mathrm{C}$ series versus reduction time, calculated from eq. (6) and the $\rho_{t}-t$ curves.

of point defects along the a-axis at each temperature and this will be discussed in section V.

\section{Discussion}

\section{Diffusion coefficient}

As the concentration of point defects introduced by reduction seems to be very small $^{(1)(2)}$, it is considered that the diffusion coefficient of point defects does not depend on their concentration. As shown in Figs. 4 and 5 , however, the $D$ values of $A$ and $B$ series computed in accordance with eq. (6) changes with time $t$, but the $D$ value in $\mathrm{C}$ series is independent of $t$ except for an early stage at each temperature. These phenomena may be due to the fact that the results of $\mathrm{A}$ and $B$ series are influenced by the contamination from diffusion pump oil, but not so in $\mathrm{C}$ series.

At a certain temperature in $B$ series, the $D$ value increases with $t$ after passing a minimum value. This is attributed to the increase of the amount of contamination with increase in reduction time. On the other hand, in A series using diffusion pump without traps, the amount of the adsorbed molecules is very large in comparison with B series, so that the increase in $D$ at $800^{\circ} \mathrm{C}$ in $\mathrm{A}$ series is considerably larger than that at $900^{\circ} \mathrm{C}$ in $\mathrm{B}$ series, as shown in Fig. 4.

As the amount of contamination molecules on the surface increases, the difference in the concentration between the surface and the inner part also increases and the diffusivity to the inner part may be facilitated. The dominant components of contamination are $\mathrm{H}$ and $\mathrm{C}$ atoms ${ }^{(16)}$, and they can diffuse into the specimen very easily because of their small ionic radii. For these reasons, the $D$ values increase with reduction time in $A$ and $B$ series.

\section{Comparison of specific resistivities}

There is a large difference in specific resistivity between $A$ and $B$ series and $C$ series. Table 3 shows the constant equilibrium values of specific resistivities. Under a constant vacuum, the concentration of point

Table 3 Comparison of $\rho_{\infty}$ values by temperatures, atmospheres and $2 h$.

\begin{tabular}{|c|c|c|c|}
\hline$\stackrel{T}{\left({ }^{\circ} \mathrm{C}\right)}$ & Series & $\begin{array}{r}2 h \\
(\mathrm{~cm})\end{array}$ & $\stackrel{\rho_{\infty}}{(\mathrm{ohm}-\mathrm{cm})}$ \\
\hline $\begin{array}{r}800 \\
900 \\
1000 \\
1100 \\
1200 \\
800 \\
900 \\
1000 \\
1000 \\
1100\end{array}$ & $\begin{array}{l}\mathbf{A} \\
\mathbf{B} \\
\mathbf{B} \\
\mathbf{B} \\
\mathbf{B} \\
\mathrm{C} \\
\mathrm{C} \\
\mathrm{C} \\
\mathrm{C} \\
\mathrm{C} \\
\mathrm{C}\end{array}$ & $\begin{array}{l}0.0820 \\
0.0774 \\
0.0812 \\
0.0766 \\
0.0826 \\
0.0491 \\
0.0670 \\
0.0936 \\
0.0571 \\
0.1614\end{array}$ & $\begin{array}{l}0.3672 \\
0.3932 \\
0.3602 \\
0.5745 \\
0.5845 \\
1.1207 \\
0.6564 \\
1.3924 \\
0.1709 \\
1.4684\end{array}$ \\
\hline
\end{tabular}

defects should increase with the rise of reduction temperature, and then the $\rho_{\infty}$ value should become smaller. But this is not the case in $C$ series. For example, the $\rho_{\infty}$ value of the specimen with thickness $2 h=0.936 \mathrm{~mm}$ reduced at $1000^{\circ} \mathrm{C}$ is $1.392 \mathrm{ohm}-\mathrm{cm}$, while the $\rho_{\infty}$ value of the specimen with $0.491 \mathrm{~mm}$ as $2 h$ reduced at $800^{\circ} \mathrm{C}$ is $1.121 \mathrm{ohm}-\mathrm{cm}$. This is incosistent with the above consideration. The origin of this anomaly remains unaccounted for. But it is quite certain that the thickness of specimens has a great influence. But even though the thickness of specimens is taken into consideration, the $\rho_{t}$ values in A, B series are distinctly smaller than those in $C$ series. This indicates that the influence of atmospheres on reduction is much larger than that of the thickness of specimens.

There are two factors to be considered in the reduction atmosphere, i.e. (1) the vacuum condition and (2) contamination. If the former is a dominant factor, the $\rho_{\infty}$ values in $\mathrm{C}$ series must be smaller than those in $A$ and $B$ series because of the higher vacuum in $C$ series. But in the experiment, the $\rho_{\infty}$ values in $C$ series are larger than those in $A$ and $B$ series. Therefore the effect of contamination is responsible for this result.

(16) R.K.Hart, T.F.Kassner and J.K. Maurin : Phil. Mag., 21 (1970), 453 . 
In $A$ and $B$ series, the contamination molecules may diffuse into the specimen and release electrons as donor centers, giving a low resistivity.

From the above considerations, it may be concluded that the reduction in $A$ and $B$ series is influenced by contamination from diffusion pump oil, but $\mathrm{C}$ series is perfectly free from contamination.

\section{C series}

Equation (6) is derived on the assumption that only one kind of point defects is responsible for diffusion. If contamination molecules adsorbed on the surfaces diffuse into the specimen, the condition is very complicated. Therefore, the results in A and B series can hardly be analyzed quantitatively.

In order to detect real behaviours of point defects in the reduced rutile, contamination must be eliminated by using an ion pump used instead of a diffusion pump even if liquid nitrogen traps are equipped.

It is certain that the results in $\mathrm{C}$ series show real behaviours of point defects in the reduced rutile. But these results are not necessarily consistent with theory. For instance, a theoretical $\rho_{t}-t$ curve shown in Fig. 2 which is derived from eq. (6) deviates from the experimental one at the early stage of reduction. The following three reasons are considered for this deviation.

(1) Because of the early stage of reduction, the three assumptions described in section II may not be realized. The assumption (2) especially may hardly be realized.

(2) In the very early stage, the defect concentration in the inner part of the specimen is very small, so the electronic current flows preferentially near the surface.

(3) There are other kinds of point defects in the reduced rutile which diffuse very fast with small activation energies in the early stage of reduction. Perhaps the reasons (1) and (2), especially (2), may dominantly contribute to this deviation.

In conclusion, real behaviours of point defects in the reduced rutile can be investigated in $\mathrm{C}$ series free from contamination. The relationship of $\log D$ versus $(1 / T)$ in $\mathrm{C}$ series is obtained as in eq. (7) and plotted in Fig. 6.

$$
D=91 \exp [-(2.42 \pm 0.33)(\mathrm{eV}) / k T]\left(\mathrm{cm}^{2} / \mathrm{sec}\right) .(7)
$$

Equation (7) is considered to represent the diffusion coefficient of point defects along the a-axis.

(17) R. Haul and G. Dumbgen : J. Phys. Chem. Solids., 26(1965), 1. (18) E. Iguchi and K. Yajima to be published in J. Phys. Soc. Japan.

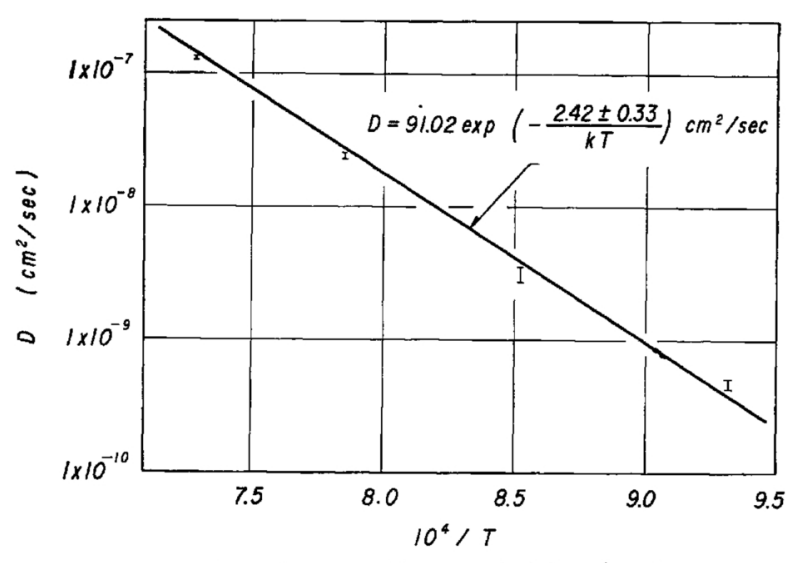

Fig. 6 The relation of $D$ versus $(1 / T)$ in $C$ series.

It is very interesting to note that the activation energy along the a-axis, $2.42 \mathrm{eV}$, is very close to the activation energy of oxygen ions $2.6 \mathrm{eV}$, which diffuse by the vacancy mechanism in rutile ${ }^{(17)}$. For the research of point defects in reduced rutile, it also needs to investigate the behaviour along the c-axis, which are now in progress at our laboratory ${ }^{(18)}$.

\section{Summary}

Rutile single crystals were reduced in three differeent vacuum atmospheres. The following conclusions are obtained.

(1) Rutile specimens reduced in a vacuum system using a diffusion pump equipped with liquid nitrogen traps are contaminated with vapour of diffusion pump oil and the contamination influences the kinetic behaviours of point defects.

(2) Specimens reduced in a vacuum system using an ion pump are completely free from the contamination. The activation energy of diffusion of point defects in rutile reduced by this method is very close to that of oxygen ions which diffuse by the vacancy mechanism.

\section{Acknowledgments}

The authors wish to express their appreciation to Prof. R. Wada, Yokohama National University, for his encouragement and helpful discussion. Thanks are also due to Mr.S. Takahashi, National Research Institute for Metals, for cutting single crystals to slices. 